\author{
ENSV TEADUSTE AKADEEMIA TOIMETISED. XVII KOIDE \\ KEEMIA * GEOLOOGIA. 1968, Nr. 2 \\ ИЗВЕСТИЯ АКАДЕМИИ НАУК ЭСТОНСКОИ ССР. ТОМ ХVII \\ Химия * ГЕОлогия. 1968, № 2
}

K. ХАБНХТ

\title{
ВОПРОСЫ ЭКОНОМИКИ ИСПОЛЬЗОВАНИЯ ЗАПАСОВ ПОЛЕЗНЫХ ИСКОПАЕМЫХ
}

Проблема экономически оптимального нспользования запасов недр возникает прн распределении исследуемых запасов на балансовые и забалансовые, а также при оценке допустимости дополнительных потерь полезных ископаемых, вызываемых в действующих рудниках изменением системы разработки и механизацией горных работ.

Ниже предлагается методика определения экономически наиболее оправданного варианта использования запасов шахтного поля, основывающаяся на сопоставлении всех последующих текущих и капитальных затрат, вызванных разными варнантами использования данных запасов и необходимых для получения эквивалентного колнчества конечного продукта.

Статья состоит из двух частей. Первая посвящена экономической оценке потерь полезного ископаемого в действующем руднике в условиях постоянного объема годовой добыни. Во второй приводится методика определения нанболее оптимальной степени использования запасов нового шахтного поля.

В данной статье не рассматривается случай наиболее полного использования запасов с целью повышения выпуска продукции уже существующей шахты. Для обоснования численных величин использованных в статье коэффициентов требуется еще самостоятельное исследование экспертных ощенок и статистического материала.

\section{1. Методика экономической оценки потерь запасов на эксплуатируемом руднике}

Рассмотрим случай, когда на руднике возможны два варианта добычных работ. При внедрении варианта I разрабатываемые запасы обеспечивают заданную руднику годовую добычу полезного ископаемого еще на $T_{1}$ лет с себестоимостью единицы продукции в базовом году $r_{1}$ руб. При варианте II эксплуатируются бо́льшие запасы - дополнительно используются и те запасы, которые при добыче или обогащении требуют более высоких затрат и остаются поэтому в варианте I неиспользованными и навсегда потерянными для народного хозяйства. Если в варианте II суммарная потребительная стоимость годовой добычи остается неизменной, срок службы шахты удлиняется вследствие увеличения балансовых запасов на $T_{2}-T_{1}=\Delta T$ лет. Себестоимость единицы продукции эквивалентной потребительной стоимости составит теперь в базовом году $r_{2}$, причем $r_{2}>r_{1}$. Но, с другой стороны, при экономическом сравнении вариантов должно быть учтено удлинение срока службы рудника в варианте II.

Для оценки эффекта от увеличения срока эксплуатации рассмотрим изменение во времени средних в данном бассейне затрат по добыче и 
обогащению. Средняя себестоимость единицы продукции данной потребительной стоимости в базовом году $R$ в последующих годах изменится под влиянием двух факторов:

1) в результате технического прогресса в горной промышленности, ежегодное влияние которого на производственные затраты учитывает коэффициент $\mu(\mu<1,0)$;

2) вследствие ухудшения условий горных работ и качества добываемых запасов, обусловленного отработкой более богатых и легче разрабатываемых месторождений. Влияние этого фактора на производственные затраты учитывает коэффициент $\varepsilon(\varepsilon>1,0)$.

Если рассматривать технический прогресс и ухудшение во времени горнотехнических условий как непрерывный процесс, то среднюю себестоимость единицы продукции данной потребительной стоимости $t$ лет после базового года можно выразить в виде

$$
R_{t}^{\prime}=R \mu^{t} \varepsilon^{t} .
$$

Для того чтобы сравнить относительно высокие при варианте II производственные затраты первых лет с относительно низкими затратами в более позднем периоде (что влечет за собой соответствующие изменения прибыли), необходимо производственные затраты всех периодов привести в сопоставимость с затратами базового года.

Считается, что прибыль более позднего периода имеет меньший экономический вес, так как общественный производственный процесс выступает в качестве расширенного воспроизводства. Исходя из этого, коэффициент приведения $\gamma(\gamma<1,0)$ должен учитывать средний размер ежегодного роста национального дохода.

Средняя приведенная себестоимость единицы продукции через $t$ лет после базового года будет равна:

$$
R_{t}=R \mu^{t} \varepsilon^{t} \gamma^{t}=R \alpha^{t},
$$

где $\alpha$ - коэффициент, учитывающий изменение во времени средней по бассейну приведенной себестоимости $(\alpha<1,0)$.

В случае варианта I данный рудник эксплуатируется в период $O T_{1}$, причем приведенная себестоимость единицы продукции за каждый входящий в период $O T_{1}$ год $t$ выражается в виде

$$
r_{1}^{(t)}=r_{1} \mu^{t} \gamma^{t}=r_{1} \beta^{t},
$$

где $\beta$ - коэффициент, учитывающий изменение во времени приведенной себестоимости данного рудника $(\beta<\alpha<1,0)$.

В данном случае не учтен коэффициент $\varepsilon$, поскольку предполагалось, что ухудшение условий горных работ проявляется лишь при переходе к эксплуатации нового рудника и не наблюдается при эксплуатации одной и той же шахты.

При варианте II приведенная себестоимость единицы продукции как в периоде $O T_{1}$, так и в последующем перноде $\Delta T$ составляет

$$
r_{2}^{(t)}=r_{2} \beta^{t} .
$$

Суммарный рост приведенной себестоимости на единицу продукции (и соответствующее уменьшение приведенной прибыли) при переходе с варианта I на вариант II в периоде $O T_{1}$ выражается в виде

$$
r_{2} \int_{0}^{T_{1}} \beta^{t} d t-r_{1} \int_{0}^{T_{1}} \beta^{t} d t=\left(r_{2}-r_{1}\right) \frac{\beta^{T_{1}}-1}{\ln \beta} .
$$


В течение следующего периода $\Delta T$ при варианте I запасы данного шахтного поля уже исчерпываются и начинается эксплуатация новой шахты (рис. 1). В случае варианта II работа в рассматриваемой шахте

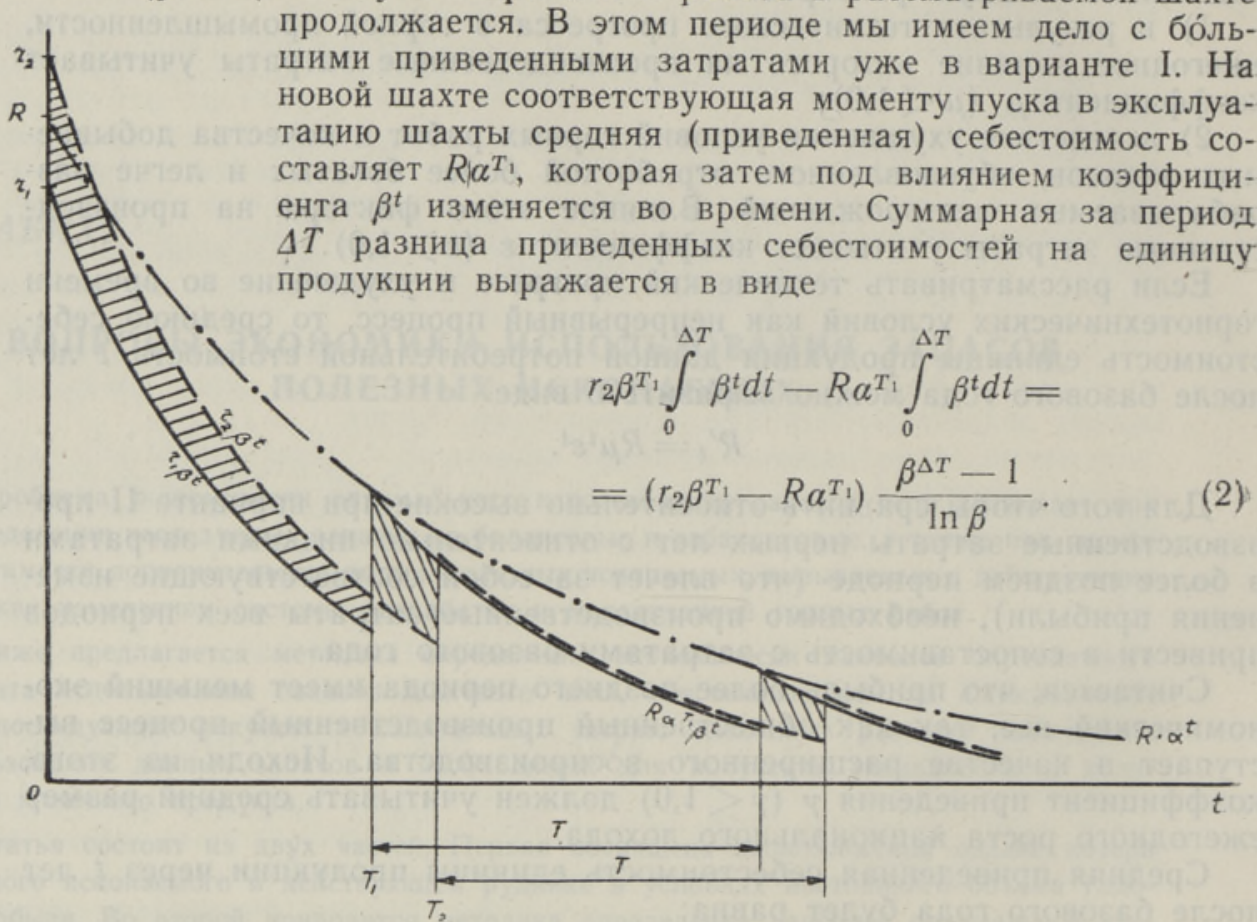

Рис. 1. Изменение приведенной себестоимости во времени по вариантам.

В следующем периоде, когда как при варианте I, так и при варианте II эксплуатируется одна и та же новая шахта, приведенные себестоимости равны. Различие между ними снова проявляется тогда, когда в случае варианта I истекает стандартный срок эксплуатации шахты $(T)$ и в строй вводится опять новая шахта. При варианте II до истечения срока эксплуатации шахты остается еще $\Delta T$ лет. В течение таких периодов $\Delta T$, повторяющихся через каждые $T-\Delta T$ лет, приведенная себестоимость при варианте II будет ниже. Суммарная разница приведенных себестоимостей на единицу продукции за все такие промежутки времени $\Delta T$ выразится в виде

$$
\begin{aligned}
& \left(R a^{T_{1}} \beta^{T}-R a^{T_{1}+T}\right) \int_{0}^{\Delta T} \beta^{t} d t+\left(R \alpha^{T_{1}+T} \beta^{T}-R \alpha^{T_{1}+2 T}\right) \int_{0}^{\Delta T} \beta^{t} d t+\ldots= \\
& =R \frac{\beta^{\Delta T}-1}{\ln \beta}\left(\alpha^{T_{1}} \beta^{T} \frac{1}{1-\alpha^{T}}-\frac{a^{T_{1}+T}}{1-\alpha^{T}}\right)=R \frac{\alpha^{T_{1}}\left(\beta^{T}-\alpha^{T}\right)\left(\beta^{\Delta T}-1\right)}{\left(1-\alpha^{T}\right) \ln \beta} .
\end{aligned}
$$

При этом, поскольку $a<1,0$, можно применить формулу суммы бесконечно убывающей геометрической прогрессии.

Суммируя разницы приведенных себестоимостей вариантов I и II во всех промежутках времени (формулы (1)-(3)), получаем суммар- 
ный рост приведенной себестоимости на единицу продукции, характеризующий внедрение варианта II по сравнению с вариантом I:

$$
\begin{aligned}
L & =\left(r_{2}-r_{1}\right) \frac{\beta^{T_{1}}-1}{\ln \beta}+\left(r_{2} \beta^{T_{1}}-R a^{T_{1}}\right) \frac{\beta^{\Delta T}-1}{\ln \beta}+ \\
& +R \frac{a^{T_{1}}\left(\beta^{T}-a^{T}\right)\left(\beta^{\Delta T}-1\right)}{\left(1-\alpha^{T}\right) \ln \beta}= \\
& =r_{1} \frac{1-\beta^{T_{1}}}{\ln \beta}-r_{2} \frac{1-\beta^{T_{2}}}{\ln \beta}-R \frac{\alpha^{T_{1}}\left(1-\beta^{T}\right)\left(\beta^{\Delta T}-1\right)}{\left(1-\alpha^{T}\right) \ln \beta} .
\end{aligned}
$$

По приведенной себестоимости единицы продукции вариант II следует предпочесть варианту I, если

$$
L<0 \text {. }
$$

Наряду с текущими затратами при оценке экономической эффективности более полного использования запасов полезного ископаемого необходимо проанализировать и производимые при различных вариантах капитальные вложения.

В случае варианта II явно необходимы по сравнению с вариантом I дополнительные капитальные вложения:

1) на руднике, где совместная добыча более бедного ископаемоro может потребовать дополнительных капитальных вложений главным образом для увеличения пропускной способности подземного транспорта и подъема;

2) на обогатительной фабрике, поскольку большее количество бедной руды требует увеличения пропускной способности фабрики;

3) у потребителя, если обогащенная руда остается относительно бедной.

Обозначим упомянутые выше дополнительные капитальные вложения через $\Delta K$ и допустим условно, что они производятся в базовом году (рис. 2).

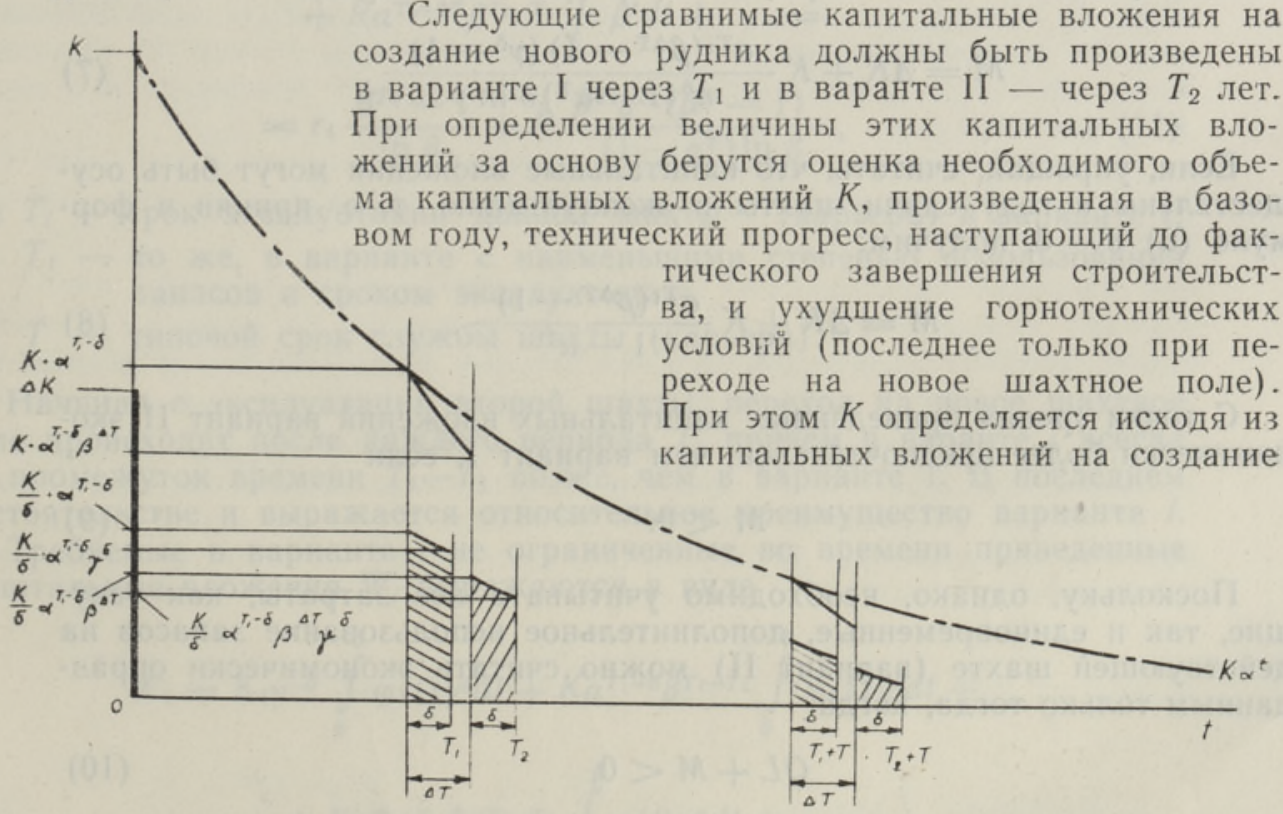

Рис. 2. Приведенные капитальные вложения по вариантам. 
шахты типовой мощности и из соотношения годовой добычи первоначальной и типовой шахт.

Далее исходим из следующих предпосылок:

1) срок капитального строительства длится $\delta$ лет, следовательно капитальное строительство должно начаться в варианте I через $T_{1}-\delta$ и в варианте II через $T_{2}-\delta$ лет;

2) распределение объема капитальных вложений в течение $\delta$ лет установлено функцией $\varphi(t)$;

3) капитальные вложения на создание новой шахты для замены выбывающей повторяются в обоих вариантах через $T$ лет, при этом в варианте II этот цикл отодвинут на $\Delta T$ лет вперед;

4) все сравниваемые капитальные вложения переведены на базовый год и, таким образом, сопоставимы.

Принимая во внимание вышеизложенное, рост суммарных капитальных вложений по варианту II в сравнении с вариантом I можно выразить следуюлцим образом:

is.

$$
\begin{gathered}
M=\Delta K+K \alpha^{T_{1}-\delta} \beta^{\Delta T} \int_{0}^{\delta} \varphi(t) \gamma^{t} d t-K \alpha^{T_{1}-\delta} \int_{0}^{\delta} \varphi(t) \gamma^{t} d t+ \\
+K a^{T_{1}+T-\delta} \beta^{\Delta T} \int_{0}^{\delta} \varphi(t) \gamma^{t} d t-K a^{T_{1}+T-\delta} \int_{0}^{\delta} \varphi(t) \gamma^{t} d t+ \\
+K a^{T_{1}+2 T-\delta} \beta^{\Delta T} \int_{0}^{\delta} \varphi(t) \gamma^{t} d t-\ldots
\end{gathered}
$$

Считая условно распределение капитальных вложений в периоде $\delta$ равномерным $\left(\varphi(t)=\frac{1}{\delta}\right)$, интегрируя и применяя формулу суммы бесконечно убывающей геометрической прогрессии, получим

$$
M=\Delta K+K \frac{a^{T_{1}}\left(\beta^{\Delta T}-1\right)\left(\gamma^{\delta}-1\right)}{a^{\delta}\left(1-a^{T}\right) \delta \ln \gamma} .
$$

Если, упрощая, считать, что капитальные вложения могут быть осуществлены в году сдачи шахты в эксплуатацию, т. е. приняв в формуле (6) $\delta=0$, получим:

$$
M=\Delta K+K \frac{\alpha^{T_{1}}\left(\beta^{\Delta T}-1\right)}{1-\alpha^{T}} .
$$

C точки зрения приведенных капитальных вложений вариант II экономически более предпочтителен, чем вариант I, если

$$
M<0 .
$$

Поскольку, однако, необходимо учитывать все затраты, как текущие, так и единовременные, дополнительное использование запасов на действующей шахте (вариант II) можно считать экономически опразданным только тогда, когда

$$
Q L+M<0
$$

где $Q$ - годовая добыча на данной шахте. 


\section{2. Методика определения оптимального варианта использования запасов}

Рассмотрим теперь общий случай, когда из множества вариантов $N$ нужно найти экономически лучший вариант использования запасов даћного месторождения, при условии обеспечения планового или эквивалентного ему по потребительной стоимости количества концентрата. Варианты отличаются один от другого по степени использования запасов, по эксплуатационным расходам и необходимым капитальным вложениям.

Если шахтное поле ограничено, то более полное использование запасов при плановом объеме продукции неизменного качества означает удлинение срока. эксплуатации данного шахтного поля. Такое положение в республике наблюдается на фосфоритных карьерах, где совместная с кондиционным фосфоритом выемка пластов с меньшим содержанием $\mathrm{P}_{2} \mathrm{O}_{5}$ позволила бы продлить срок службы карьера.

Если мы имеем дело с запасами, расположенными на большой территории, то более полное использование запасов означает более медленное перемещение горных работ из лучше разрабатываемой или более производительной части бассейна в менее выгодные условия. В Эстонской ССР такой случай наблюдается в сланцевом бассейне.

Рассмотрим оба случая отдельно, применив изложенную в предыдущем пункте методику и обозначения.

А. В случае шахтного поля с ограниченными запасами сумма приведенных эксплуатационных затрат за неограниченный период времени на единицу продукции по варианту $i$ выражается в виде

$$
\begin{aligned}
V_{i}=r_{i} \int_{0}^{T_{i}} \beta^{t} d t & +R \alpha^{T_{1}} \beta^{T_{i}-T_{1}} \int_{0}^{T} \beta^{t} d t+R \alpha^{T_{1}+T} \beta^{T_{i}-T_{1}} \int_{0}^{T} \beta^{t} d t+ \\
& +R \alpha^{T_{1}+2 T} \beta^{T_{i}-T_{1}} \int_{0}^{T} \beta^{t} d t+\ldots= \\
= & r_{i} \frac{\beta^{T_{i}}-1}{\ln \beta}+R \frac{\beta^{T_{i}} \varepsilon^{T_{1}}\left(\beta^{T}-1\right)}{\left(1-\alpha^{T}\right) \ln \beta}
\end{aligned}
$$

где $T_{i}$ - срок эксплуатации данной шахты (карьера) в варианте $i$;

$T_{1}$ - то же, в варианте с наименьшими степенью использования запасов и сроком эксплуатации;

$T$ - типовой срок службы шахты (карьера).

Начиная с эксплуатации второй шахты, переход на новое шахтное поле происходит после каждого периода $T$, причем в варанте $i$ всегда на промежуток времени $T_{i}-T_{1}$ позже, чем в варианте I. В последнем обстоятельстве и выражается относительное преимущество варианта $i$.

Требуемые в варианте $i$ не ограниченные во времени приведенные капитальные вложения $W_{i}$ выражаются в виде

$$
\begin{gathered}
W_{i}=K_{i} \gamma^{-\delta} \int_{0}^{\delta} \varphi(t) \gamma^{t} d t+K \alpha^{T_{1}-\delta} \beta^{T_{i}-T_{1}} \int_{0}^{\delta} \varphi(t) \gamma^{t} d t+ \\
+K \alpha^{T_{1}+T-\delta} \beta^{T_{t}-T_{1}} \int_{0}^{\delta} \varphi(t) \gamma^{t} d t+\ldots
\end{gathered}
$$


Как в формуле (6), так и здесь при оценке величины капитальных вложений $K$ исходили из капитальных вложений, необходимых для создания рудника типовой мощности и скорректированных с учетом отношения добычи создаваемого в рассматриваемом месторождении рудника к добыче рудника типовой мощности.

При выведении $W_{i}$ имелось в виду, что для внедрения в базовом году варианта $i$ капитальные вложения требуются уже в течение предшествующих базовому году $\delta$ лет. Вкладываемые в этом периоде средства также приведены к базовому году. Для пояснения формулы следовало бы еще отметить, что если начало создания одного и того же рудника предусматривается в различные сроки (напр., $T_{i}-\delta, T_{1}-\delta$ ), то в промежуток времени от $T_{1}-\delta$ до $T_{i}-\delta$ никакого ухудшения горнотехнических условий, влияющего на капитальные вложения, быть не может.

Допуская условно, что капитальные вложения в пернод строительства $\delta$ распределены равномерно $\left(\varphi(t)=\frac{1}{\delta}\right)$, получаем

$$
W_{i}=K_{i} \frac{\gamma^{\delta}-1}{\gamma^{\delta} \cdot \delta \cdot \ln \gamma}+K \frac{\beta^{T_{i}} \varepsilon^{T_{1}}\left(\gamma^{\delta}-1\right)}{a^{\delta}\left(1-a^{T}\right) \delta \ln \gamma} .
$$

Для определения экономически наиболее целесообразного варианта эксплуатации шахтного поля с ограниченными запасами необходимо найти

$$
\min _{i \in N}\left(Q V_{i}+W_{i}\right)
$$

где $Q$ - годовая добыча на данной шахте.

Б. В случае разработки пластовых месторождений крупного бассейна производительность квадратного метра пласта определяет ту скорость, с какой горные работы продвигаются в направлении запасов более низкого качества. Типовая мощность и соответствующий оптимальный срок эксплуатации шахты обеспечиваются выбором величины шахтного поля. В отличие от случая $A$, здесь при всех вариантах использованил запасов срок эксплуатации $T$ остается постоянным, размер же шахтного поля, наоборот, изменяется. Это вносит известное изменение и в методику расчетов.

Переход в более трудные горнотехнические условия происходит тем медленнее, чем выше производительность разрабатываемого пласта. Если обозначить

$$
c_{i}=\frac{p_{1}}{p_{i}},
$$

где $p_{1}$ и $p_{i}$ - производительность пласта в варианте $i$ и взятом за основу варианте I с наименьшей степенью использования запасов, то фактор $\varepsilon^{c_{i} t}$ учитывает влияние ухудшения горнотехнических условий на среднюю себестоимость и средние капитальные вложения через $t$ лет.

В случае разработки крупного бассейна сумма не ограниченных во времени приведенных эксплуатационных затрат на единицу продукции по варианту $i$ составит

$$
\begin{aligned}
\bar{V}_{i} & =r_{i} \int_{0}^{T} \beta^{t} d t+R \beta^{T} \varepsilon^{c i T} \int_{0}^{T} \beta^{t} d t+R \alpha^{T} \beta^{T} \varepsilon^{c i T} \int_{0}^{T} \beta^{t} d t+ \\
& +R a^{2 T} \beta^{T} \varepsilon^{c i T} \int_{0}^{T} \beta^{t} d t+\ldots=r_{i} \frac{\beta^{T}-1}{\ln \beta}+R \frac{\beta^{T} \varepsilon^{c i T}\left(\beta^{T}-1\right)}{\left(1-\alpha^{T}\right) \ln \beta} .
\end{aligned}
$$


Не ограниченные во времени приведенные капитальные вложения $\bar{W}_{i}$ варианта $i$ можно выразить в виде

$$
\begin{aligned}
\bar{W}_{i} & =K_{i} \gamma^{-\delta} \int_{0}^{\delta} \varphi(t) \gamma^{t} d t+K \beta^{T-\delta} \varepsilon^{c i(T-\delta)} \int_{0}^{\delta} \varphi(t) \gamma^{t} d t+ \\
& +K \alpha^{T-\delta} \beta^{T} \varepsilon^{c i T} \int_{0}^{\delta} \varphi(t) \gamma^{t} d t+K \alpha^{2 T-\delta} \beta^{T} \varepsilon^{c i T} \int_{0}^{\delta} \varphi(t) \gamma^{t} d t+\ldots
\end{aligned}
$$

Полагая и здесь $\varphi(t)=\frac{1}{\delta}$, получаем

$$
\bar{W}_{i}=K_{i} \frac{\gamma^{\delta}-1}{\gamma^{\delta} \cdot \delta \cdot \ln \gamma}+K \frac{\beta^{T} \varepsilon^{c i T}\left(\gamma^{\delta}-1\right)\left(1-\alpha^{T}+a^{T-\delta} \beta^{\delta} \varepsilon^{c i \delta}\right)}{\beta^{\delta} \cdot \varepsilon^{c i \delta} \cdot \delta\left(1-\alpha^{T}\right) \ln \gamma} .
$$

Если работы ведутся в крупном бассейне, то для определения экономически наиболее целесообразного варианта использования запасов необходимо найти

$$
\min _{i \in N}\left(Q \bar{V}_{i}+\bar{W}_{i}\right) .
$$

\section{Пример:}

Из четырех вариантов использования запасов необходимо найти экономически наиболее целесообразный. Условия: $T=50$ лет, $\delta=4$ года, $Q=5000000 \tau, R=2,70$ руб./T, $K=41000000$ руб., $\mu=0,95, \varepsilon=1,05$, $\gamma=\frac{1}{1,06}$. Варианты использования запасов характеризуются следуюшими показателями:

\begin{tabular}{c|c|c|c|c|c|c}
\hline Варианты & $c_{i}$ & $\begin{array}{c}r_{i} \\
\text { руб./T }\end{array}$ & $\begin{array}{c}K_{i} \\
\text { тыс. руб. }\end{array}$ & $\begin{array}{c}\bar{V}_{i} \\
\text { тыс. руб. }\end{array}$ & $\begin{array}{c}\bar{W}_{i} \\
\text { тыс. руб. }\end{array}$ & $\begin{array}{c}Q \bar{V}_{i}+\bar{W}_{i} \\
\text { тыс. руб. }\end{array}$ \\
\hline 1 & 1,0 & 2,68 & 41000 & 25400 & 50100 & 177100 \\
2 & 0,9 & 2,70 & 41500 & 25300 & 49600 & 176300 \\
3 & 0,8 & 2,74 & 43000 & 25500 & 50500 & 178000 \\
4 & 0,7 & 2,77 & 45000 & 25600 & 52000 & 180100
\end{tabular}

Таким образом, экономически наиболее оправданным является вариант 2, стоящий на втором месте по степени использования запасов (производительность пласта в 1,11 раза больше, чем в основном варианте 1).

Институт экономики

Академии наук Эстонской ССР

\section{Поступила в редакцию} 6/X 1967 


\section{K. $\mathrm{HABICHT}$}

\section{KASULIKE KAEVISTE VARUDE KASUTAMISE OKONOOMIKA KUSIMUSI}

Artiklis esitatakse metoodika pōhikontseptsioon kasulike kaeviste varude majanduslikult otstarbeka kasutamisastme kindlaksmääramiseks.

Lähtutakse variantide võrdlusest, kusjuures kriteeriumiks on minimaalne taandatud ekspluatatsioonikulude ja kapitaalmahutuste summa kindlaksmääratud tarbimisväärtusega lōpp-produkti (kontsentraadi) kohta lõpmata pikas ajavahemikus.

Taandatud kulude suurust ajas mõjutavad:

1) tehniline progress mäetööstuses;

2) kaevandamistingimuste ja kasutuselevōetavate varude kvaliteedi järkjärguline halvenemine, tingituna paremate kaevandamistingimustega leiukohtade varude ammendatusest;

3). laiendatud taastootmist iseloomustav taandamistegur, mis annab hilisema perioodi tuludele ja kuludele suhteliselt väiksema majandusliku kaalu.

Artikli esimeses osas käsitletakse maavarakadude majanduslikku hindamist töötavas kaevanduses. Teises osas esitatakse üldkujul metoodika uue leiukoha varude optimaalseima kasutamisastme kindlaksmääramiseks ja tuuakse sellekohane näide.

\section{K. HABICHT}

\section{PROBLEMS OF THE ECONOMICS OF THE EXPLOITATION OF THE USEFUL MINERAL RESOURCES}

The author presents a basic concept of the method for determining an economically efficient degree of the exploitation of useful minerals.

Proceeding from a comparison of possible variants, the criterion is considered to be the minimum sum of reduced exploitation expenses and capital investments per final product (concentrate) of a fixed use value for an indefinitely long period of time.

The amount of the reduced expenses depends, in time, on the following factors:

1) the technical progress in mining industry;

2) the gradual deterioration of the mining conditions and of the quality of the exploited minerals owing to the exhaustion of the minerals deposited in sites where superior mining conditions prevail;

3 ) the reduction factor characterizing the expanded renewal of production, which attaches a comparatively smaller economic significance to the profits and expenses of a later period.

The first part of the article deals with an economic estimation of the losses of minerals in existing mines. In the second part, methods are presented, in a generalized form, for determining an optimum degree of the exploitation of useful minerals in new deposits, and an example thereof is given. 